\title{
Heat Setting of Poly(butylene terephthalate)
}

\author{
Manisha R. Mathur, Sanjeev R. Shukla, and Prafulla B. Sawant
}

Department of Chemical Technology, University of Bombay, Matunga, Bombay 400019, India

(Received October 24, 1994)

\begin{abstract}
Poly(butylene terephthalate) (PBT) yarn was subjected to heat setting at different temperatures for various time periods under taut conditions. Properties such as breaking load, critical dissolution time, dyeing with disperse dyes, etc. were evaluated and related to the changes taking place in the internal fiber structure. A temperature of about $140^{\circ} \mathrm{C}$ and a time period of about 60 seconds was found to be optimum for attaining the dimensional stability without affecting the useful textile properties of PBT filament yarn significantly. KEY WORDS Poly(butylene terephthalate) / Heat Setting / Dyeing /
\end{abstract}

Poly(butylene terephthalate) (PBT), a linear condensation homopolymer resembles in most of its physicochemical and mechanical properties to poly(ethylene terephthalate) (PET). PBT undergoes a unique reversible crystalline transformation at low levels of applied stress and, similar to PET, it also exhibits triclinic unit cell. The transition is related to a conformational change of the butylene chain from a relaxed gauche-trans-gauche to a stretched all-trans form.

Kinetics of heat treatment of PBT fibers and its microstructure during crystallization have been studied by Peszkin and Schultz ${ }^{1}$ using X-ray and birefringence techniques. Lue and Spruiell ${ }^{2}$ have studied the influence of processing conditions on structure development and the mechanical properties of PBT filaments. Kanno ${ }^{3}$ has reviewed the PBT fiber properties such as heat setting characteristics and printing performance. PBT fiber is also becoming popular as a "Carrierless Dyeable Polyester" with a number of advantages over normal carrier dyeable PET fiber. The main drawback of PBT, as against PET, is that it exhibits greater shrinkage. Since heat setting is essential to impart the dimensional stability, the present work was aimed at developing optimum heat setting conditions for PBT filament yarn in relation to its different useful properties such as dye uptake and strength.

\section{EXPERIMENTAL}

\section{Yarn}

PBT Filament yarn Finecell, with following specifications was used: BB 75 Denier/15 Filament, Type G 300 HP, supplied by Teijin Ltd., Japan.

\section{Dyes}

Foron Brilliant Orange ERLI (C.I. Disperse Orange 25) $(M W=323)$ and Foron Navy Blue S-2GLI (C.I. Disperse Blue 79) $(M W=639.92)$, both supplied by Sandoz (I) Ltd. Lyocol OI, dispersing agent was supplied by Sandoz (I) Ltd. All other chemicals were of "Laboratory Reagent" grade.

\section{A) Heat Setting of PBT Yarn}

PBT yarn samples were heat set under zero stretch (taut condition) for different time periods $(30,60$, and 90 seconds) at various temperatures between $100^{\circ} \mathrm{C}$ and $200^{\circ} \mathrm{C}$ with $10^{\circ} \mathrm{C}$ intervals.

B) Determination of Critical Dissolution Time (CDT)

The CDT values of the heat set PBT samples were determined in $1: 1::$ phenol:1,1-2,2 tetrachloroethane solvent system according to the method suggested by Galil. $^{4}$

\section{C) Determination of \% Crystallinity and Crystallite Size}

X-Ray diffraction studies were carried out using Philips PW 1729 X-ray generator coupled to PW 1710 Diffractometer Control and the plots were obtained on PM $8203 \mathrm{~A}$ on-line recorder. The sample powder was irradiated by $\mathrm{Ni}$-filtered $\mathrm{Cu}-K_{\alpha}$ radiation. The scanning range was $5-40^{\circ}$ and the speed was 0.02 degrees per second. The $\%$ crystallinity and crystallite size were then calculated. ${ }^{5}$

\section{D) Dyeing with Disperse Dyes}

Commercial disperse dyes were used for dyeing of the PBT samples in a Beaker Dyeing Machine. The dyebath contained disperse dye, acetic acid $\left(1 \mathrm{gl}^{-1}\right)$, and Lyocol OI $\left(0.5 \mathrm{~g}^{-1}\right)$. The shade dyed was $1 \%$, keeping liquor ratio 50 . The temperature of the dye bath was raised to $100^{\circ} \mathrm{C}$ and the dyeing was continued for 1 hour in a conventional manner. The dyed samples were rinsed with cold water and subjected to reduction-clearing with sodium hydrosulfite $\left(2 \mathrm{gl}^{-1}\right)$ and sodium hydroxide $\left(2 \mathrm{gl}^{-1}\right)$ at $65^{\circ} \mathrm{C}$ for 15 minutes to remove surface dye. Finally, the dyed samples were soaped, washed and dried.

The amount of dye taken up was estimated by extracting the dyed samples in chlorobenzene at $100^{\circ} \mathrm{C}$ for 30 minutes and determining the absorbance values of the extracts on Pye Unicam SP 8-400 UV/Vis Spectrophotometer at appropriate $\lambda_{\max }$.

\section{RESULTS AND DISCUSSION}

PBT filament yarn Finecell was heat set under taut condition and the effect of heat setting on various fiber properties was analyzed. Both time and temperature of heat setting were found to influence the strength of PBT yarns. The results plotted in Figure 1 indicate that with 


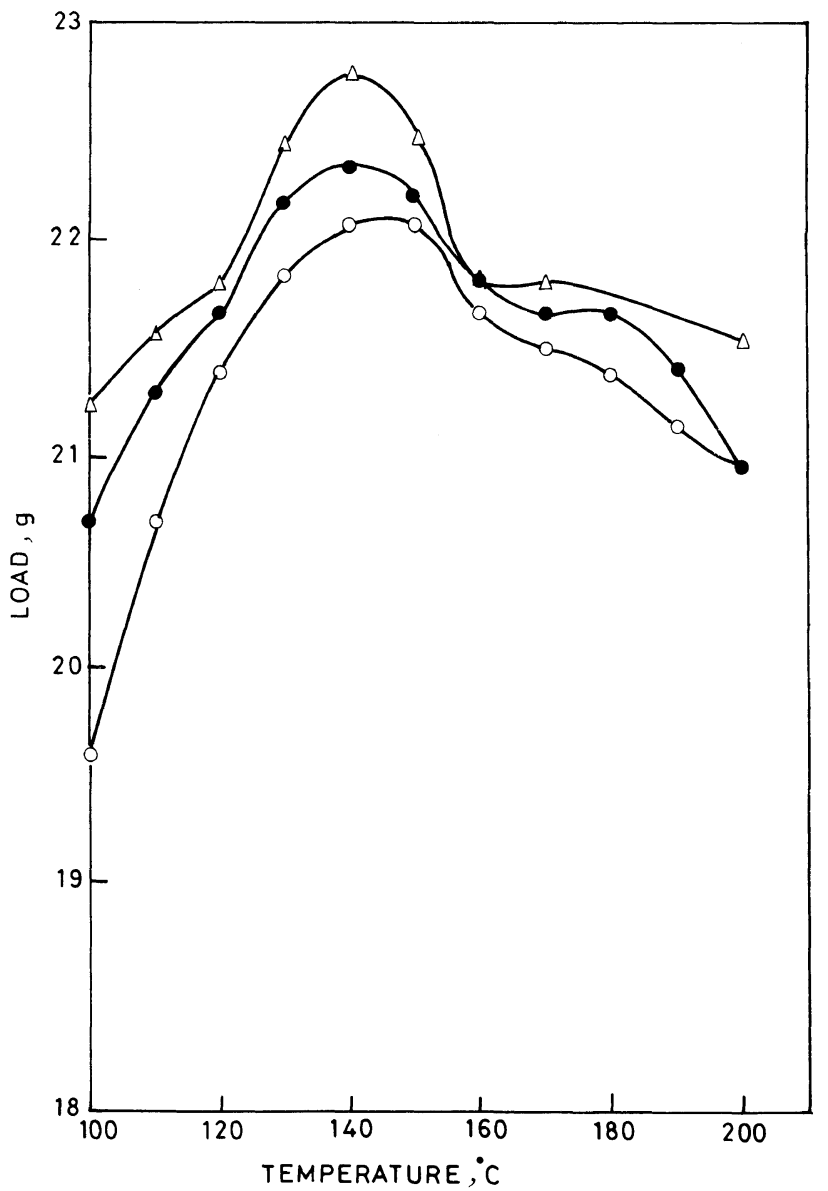

Figure 1. Plot of breaking load $v s$. heat setting temperature: $-\bigcirc-$, 30 seconds; $-\longrightarrow, 60$ seconds; $-\triangle-, 90$ seconds.

rise in temperature of heat setting upto $140^{\circ} \mathrm{C}$, the breaking load of PBT yarn increased gradually and further decreased upto $200^{\circ} \mathrm{C}$ at all the three heat setting time periods.

From the plots in Figure 2 it may be observed that upto $140-150^{\circ} \mathrm{C}$ temperature of heat setting, the CDT values increased only marginally and thereafter a very large increase in the CDT values with rise in temprature of heat treatment upto $200^{\circ} \mathrm{C}$ took place.

The data in Table I indicates that the heat setting temperature of $100^{\circ} \mathrm{C}$ decreased the $\%$ crystallinity of PBT insignificantly at any duration of treatment. At a treatment temperature of $150^{\circ} \mathrm{C}$, the $\%$ crystallinity decreased considerably and at $200^{\circ} \mathrm{C}$ it registered a further substantial decrease.

As regards the crystallite size, as observed for the two different planes (010) and (100) of the triclinic crystal of PBT, the heat setting at $100^{\circ} \mathrm{C}$ slightly decreased it in (010) plane as compared to the original unheatset sample. In the (100) plane, however, the dimensions remained the same. The $(010)$ plane lies in a direction parallel to the fiber axis in which the molecular chains, and hence the crystallites, are oriented. With further rise in temperature of heat setting upto $200^{\circ} \mathrm{C}$, however, the crystallite size increased substantially in both the planes. Heat setting at $200^{\circ} \mathrm{C}$ increased the crystallite size in the $(010)$ plane to similar levels at both the treatment periods of 30 and 90 seconds.

The heat setting treatment causes many changes in the internal structure of the fiber which are associated

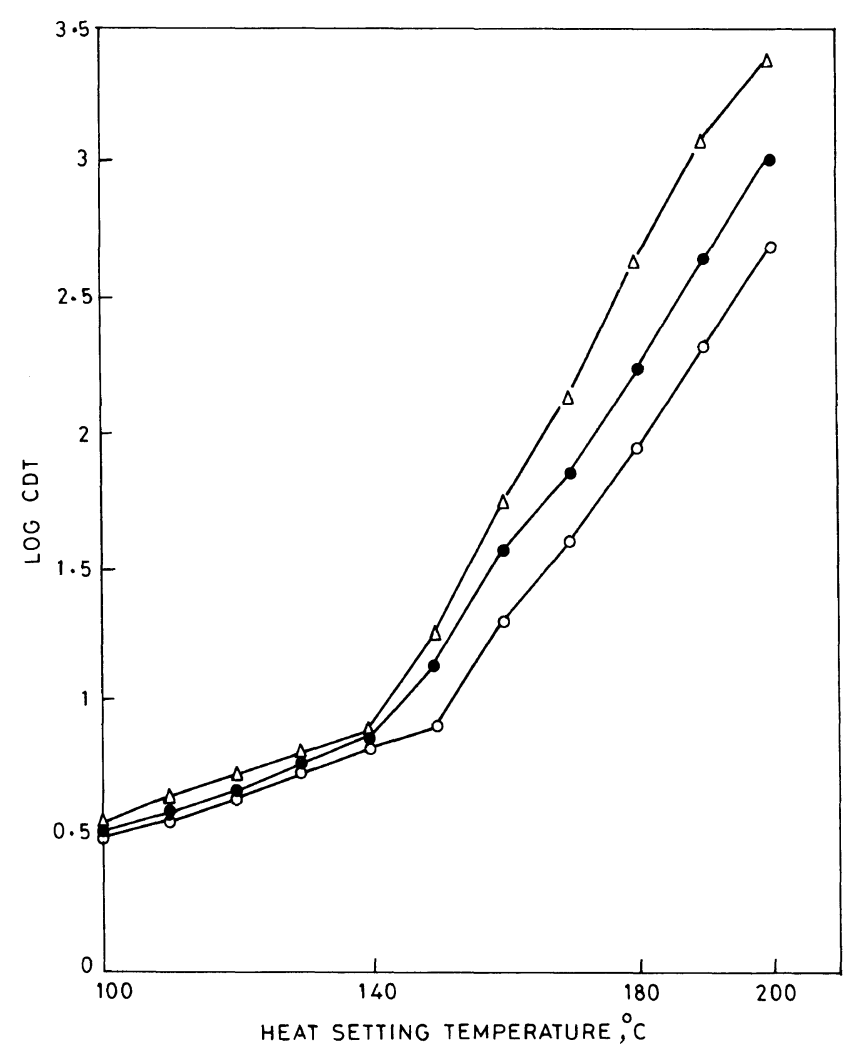

Figure 2. Plot of log critical dissolution time (CDT) i's. heat setting temperature: $-\bigcirc-, 30$ seconds; $-\bigcirc-, 60$ seconds; $-\triangle-, 90$ seconds.

with mobilization, rearrangement and stabilization of the chain molecules in the new conformation which is more stable thermodynamically. By the process of heat setting, majority of the stresses present in the fine structure of the fiber are removed thereby imparting dimensional stability to it. The quantum and the nature of these changes are dependent on the time and temperature conditions of heat setting as well as on the history of fiber manufacture.

In the heat setting temperature range of $100-140^{\circ} \mathrm{C}$, the transformation of small crystallites to the real big crystallites is extremely less and it is a temperature region which predominantly orients the existing crystallites along the fiber axis. It is well known that the breaking load increases with orientation of crystallites along the fiber axis. This phenomenon caused increase in the breaking load with rise in heat setting temperature upto $140^{\circ} \mathrm{C}$. At higher heat setting temperatures above $140^{\circ} \mathrm{C}$, the tendency of smaller crystallites to melt enhances rapidly thereby providing a very high degree of freedom to the chain molecules to allow them to come closer to each other and form bigger crystallites. Also, crystal perfection takes place at the boundary layers of the crystalline and amorphous phases. Due to this, the amorphous portion in the fiber structure virtually tends to increase thereby decreasing the breaking load.

The CDT values showed a marginal increase in the heat setting temperature range of upto $140^{\circ} \mathrm{C}$. Such changes have been reported earlier in the case of PET fibers by Groeninckx et al. ${ }^{6,7}$ The linear relationship between $\log$ CDT and the crystallite size also showed a sudden break at a temperature of about $140^{\circ} \mathrm{C}$ (Figure 
Table I. Effect of heat setting conditions on crystallinity and crystallite size of PBT yarn ${ }^{\mathrm{a}}$

\begin{tabular}{|c|c|c|c|c|c|c|}
\hline \multirow{3}{*}{$\begin{array}{c}\text { Heat setting } \\
\text { temp } /{ }^{\circ} \mathrm{C}\end{array}$} & \multirow{2}{*}{\multicolumn{2}{|c|}{ Crystallinity of sample heat set $/ \%$}} & \multicolumn{4}{|c|}{ Crystallite size of sample heat set $/ \AA$} \\
\hline & & & \multicolumn{2}{|c|}{$30 \mathrm{~s}$} & \multicolumn{2}{|c|}{$90 \mathrm{~s}$} \\
\hline & $30 s^{b}$ & $90 \mathrm{~s}^{\mathrm{c}}$ & $D_{010}$ & $D_{100}$ & $D_{010}$ & $D_{100}$ \\
\hline 100 & 77.65 & 76.80 & 50.44 & 28.09 & 53.80 & 29.12 \\
\hline 150 & 70.48 & 69.78 & 100.87 & 62.66 & 114.80 & 80.95 \\
\hline 200 & 57.52 & 52.12 & 161.50 & 108.67 & 161.40 & 116.46 \\
\hline
\end{tabular}

a Control PBT: crystallinity $/ \%=77.40$; crystallite size $/ \AA, D_{010}=62.07 ; D_{100}=27.15 . D_{010}=$ crystallite size in the direction normal to (010) plane; $D_{100}=$ crystallite size in the direction normal to (100) plane. ${ }^{\text {b.c }}$ Heat setting time.

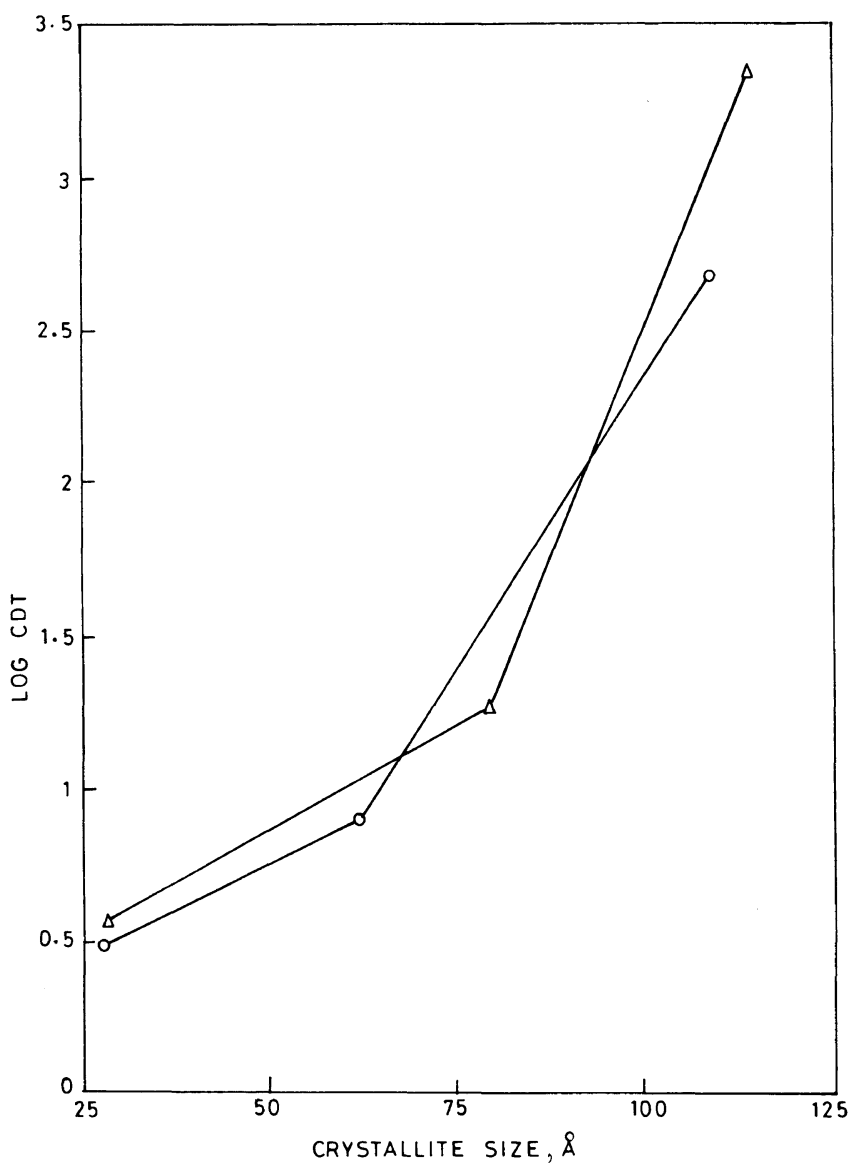

Figure 3. Plot of $\log$ CDT $v$ s. crystallite size in the direction normal to (100) plane: $-\mathrm{O}-30$ seconds; $-\triangle-90$ secons.

3). Beckmann ${ }^{8}$ had an opinion that upto this point nucleation or the formation of tiny crystals takes place and that after this specific point the conditions are suitable for sudden growth in the crystal size. The larger crystals take more time to dissolve than the smaller ones.

The heat set PBT samples were subjected to dyeing with two disperse dyes having different molecular weights, using the conventional method of dyeing. The plots of absorbance shown in Figures 4 and 5, for the dyeings with C.I. Disperse Orange 25 (low molecular weight $=323$ ) and C.I. Disperse Blue 79 (high molecular weight $=640$ ), respectively, indicate that the dye uptake reached a minimum for a sample heat set at about $140^{\circ} \mathrm{C}$ and then increased with further rise in the heat setting temprature for both the dyes. The overall dye uptake values were considerably low for the high molecular weight dye due to the hindrance caused to the bulkier

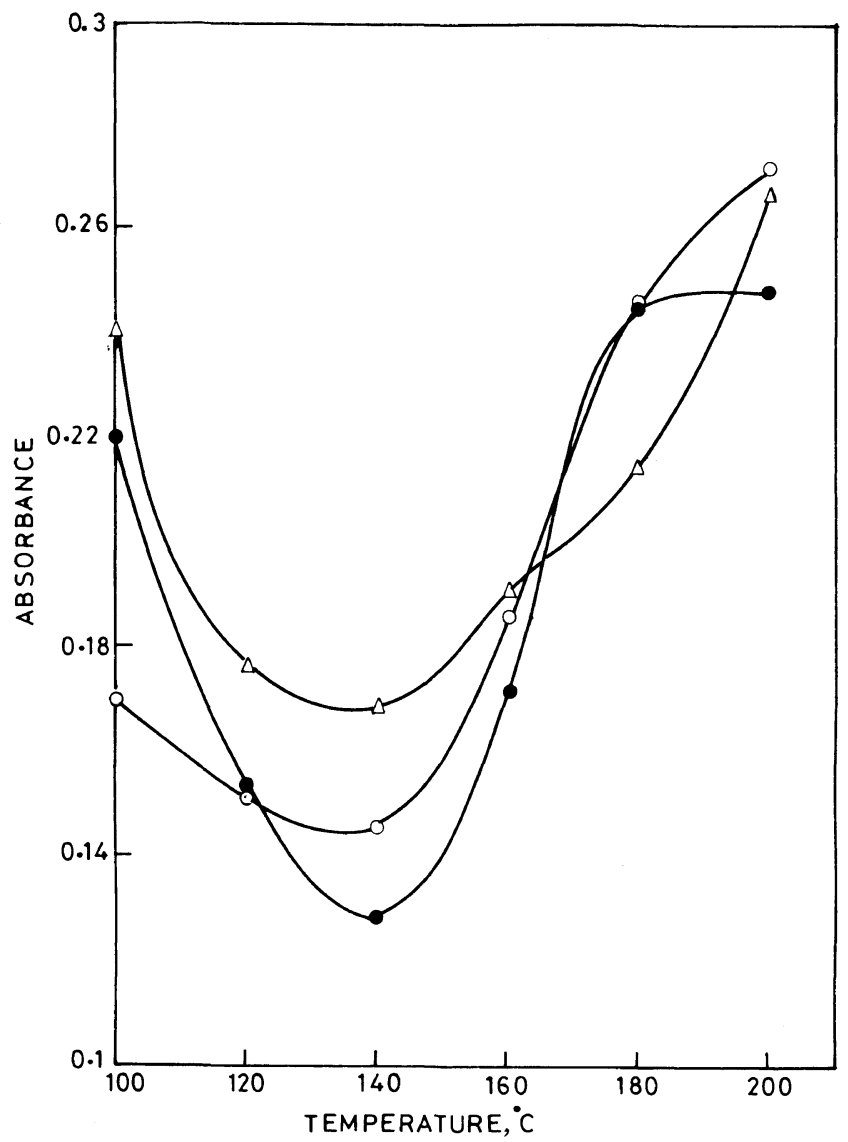

Figure 4. Plot of absorbance $v$ s. heat setting temperature for C.I. Disperse Orange 25: $-\bigcirc-, 30$ seconds; $-\bigcirc-, 60$ seconds; $-\triangle-$, 90 seconds.

dye molecules to penetrate the fiber structure.

In the initial range of heat setting, as shown in Table I, the $\%$ crystallinity has decreased and the crystallite size has increased with temperature. Generally, with a decrease in crystallinity, the dye uptake should show an increase as the accessibility of the fiber is supposed to increase. Figures 4 and 5, however, show a decrease in the dye uptake (as measured by absorbance) of treated samples. This may be attributed to the fact that the value of $\%$ crystallinity decreases due to reorganization of structure in which the smaller crystallites tend to orient themselves along the fiber axis and in the process of doing so increase the crystallite size. The dye is taken up by the fiber in the amorphous region as well as on the surface of the crystallites. With increase in the crystallite size, the surface area decreases and this factor predominantly decreases the dye uptake. Therefore, on 


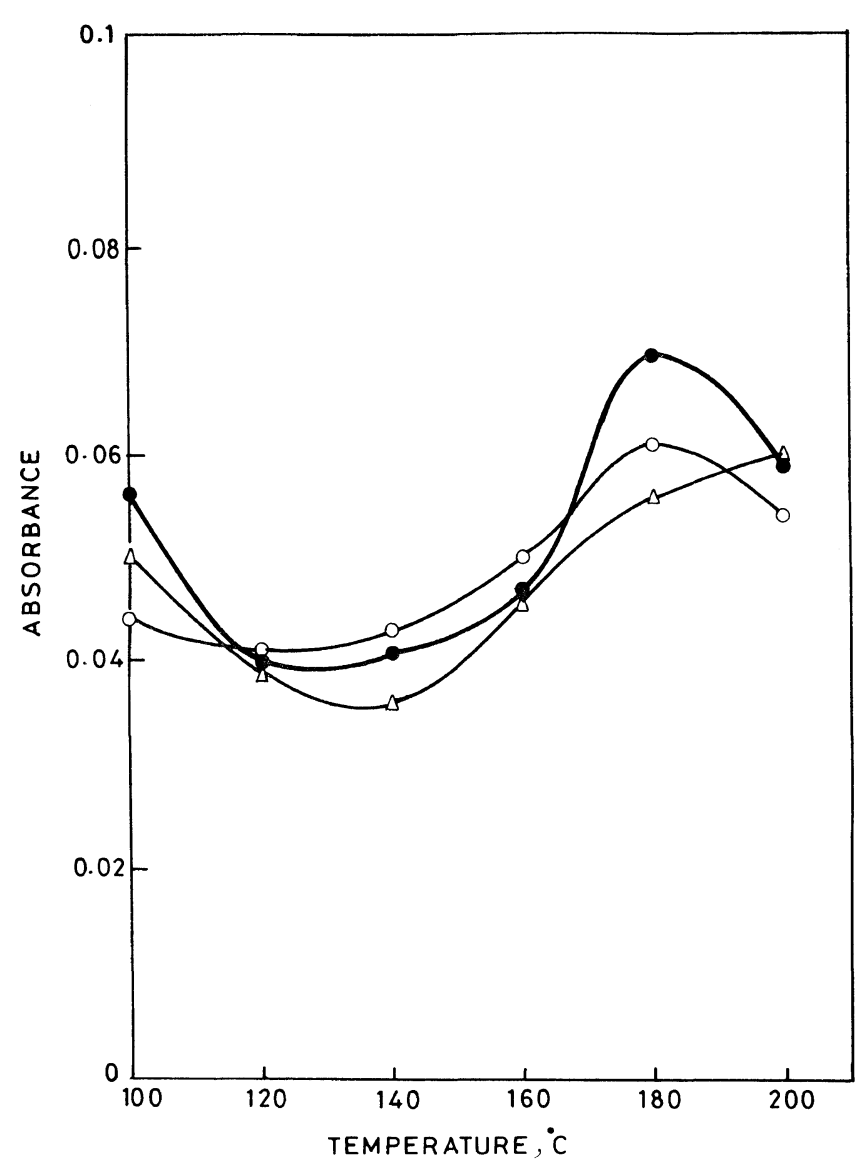

Figure 5. Plot of absorbance vs. heat setting temperature for C.I. Disperse Blue 79: $-\bigcirc-, 30$ seconds; $-\bigcirc-, 60$ seconds; $-\triangle-90$ seconds.

the whole, the effect appears to decrease the dye uptake of samples heat treated upto $140^{\circ} \mathrm{C}$. Thus, the dyeing studies also support the contention that the changes taking place in the fiber structure with the changes in heat setting temperature are of different nature upto $140^{\circ} \mathrm{C}$ and thereafter.

Since the PBT fibers are dyed only at boil, in contrast to PET fibers which are dyed at a much higher temperature of $130^{\circ} \mathrm{C}$, the heat set PBT yarns were subjected to treatment in boiling water for 1 minute under slack condition to observe their dimensional stability. These results are given in Table II. Thus, after the taut heat setting at $120^{\circ} \mathrm{C}$ for 60 seconds or more, no shrinkage took place indicating that the yarn attained the
Table II. Effect of heat setting conditions on shrinkage of PBT in boiling water

\begin{tabular}{cccc}
\hline \multirow{2}{*}{$\begin{array}{c}\text { Heat setting } \\
\text { temp } /{ }^{\circ} \mathrm{C}\end{array}$} & \multicolumn{3}{c}{ Shrinkage of PBT heat set/\% } \\
\cline { 2 - 4 } & $30 \mathrm{~s}^{\mathrm{a}}$ & $60 \mathrm{~s}^{\mathrm{b}}$ & $90 \mathrm{~s}^{\mathrm{c}}$ \\
\hline 100 & 6.25 & 5.56 & 5.70 \\
110 & 3.57 & 2.17 & 2.08 \\
120 & 1.07 & 0.00 & 0.00 \\
130 & 0.00 & 0.00 & 0.00 \\
140 & 0.00 & 0.00 & 0.00 \\
\hline
\end{tabular}

a, b, c Heat setting time.

dimenional stability at and above the heat setting temperature of $120^{\circ} \mathrm{C}$.

Heat setting should be carried out at a temperature at which the dimensional stability is fully achieved by a synthetic thermoplastic fiber. It may be observed that at $120^{\circ} \mathrm{C}$ and $140^{\circ} \mathrm{C}$ for 90 seconds treatment although the dye uptake values do not differ significantly, the rise in breaking load is significant. Also, after about $140^{\circ} \mathrm{C}$, the fiber starts undergoing major changes in the fine structure as indicated by the crystallite size and CDT values.

Hence, taking into account all the parameters related to the changes in fine structure, it is suggested that the heat setting be carried out at about $140^{\circ} \mathrm{C}$ for a duration of about 60 seconds.

Acknowledgment. The authors gratefully acknowledge the financial support given by the Council of Scientific and Industrial Research for conducting this project.

\section{REFERENCES}

1. P. N. Peszkin and J. M. Schultz, J. Polym. Sci., Polym. Phys. Ed., 24, 2591 (1980).

2. F. Lu and J. Spruiell, J. Appl. Polym. Sci., 31, 1595 (1980).

3. K. Kanno, Senshoku Kogyo, 33, 428 (1985) [Chem. Abstr., 104, 169949 (1986)].

4. F. Galil, Text. Res. J., 43, 615 (1973)

5. B. R. Manjunathan, A. Venkataraman, and S. Thomas, J. Appl. Polym. Sci., 17, 1091 (1990).

6. G. Groeninckx and H. Reynaers, J. Polym. Sci. (Phys.), 18, 1325 (1980).

7. F. Fontaine, J. Ledent, G. Groeninckx, and H. Reynaers, Polymer, 23, 185 (1982).

8. W. Beckmann and Kl. Langheinrich, Melliand Textilber., 51, 316 (1970). 